\title{
De la estética subjetiva a la condición interpretativa: entre Kant y Heidegger*
}

\author{
Enrique Rodríguez Pérez ${ }^{* *}$
}

Recibido: 7 de octubre de 2009 • Aprobado: 11 de noviembre de 2009

\section{Resumen}

Al establecer una relación entre la estética kantiana y la perspectiva hermenéutica heideggeriana se percibe un replanteamiento de la concepción del mundo y del ser humano que permite establecer semejanzas y contrastes. Las reflexiones sobre el arte en Kant, en la Crítica del Juicio, dejan ver fisuras en todo el sistema: los sentimientos de lo bello y lo sublime al poner en juego las facultades desestabilizan el sistema. Desde la mirada de Heidegger, estas fisuras llevan al giro interpretativo. El mundo se constituye en una relación distinta, sin sujeto, como relación entre cielo y tierra, divinidades y mortales. El ser humano, que ya no es sujeto, se sostiene en su propia mortalidad como evento de ausencia, sin fundamentos. Este es un modo del desplazamiento histórico de la modernidad racional al pensamiento posmetafísico.

Palabras clave: estética, hermenéutica, arte, existencia.

\footnotetext{
* Este artículo de reflexión, surgió de las discusiones en el Grupo Historia y Literatura, en la línea de Teoría Literaria y Literatura Comparada de la Universidad Nacional de Colombia, Departamento de Literatura.

** Profesor asociado del Departamento de Literatura, Universidad Nacional de Colombia. Correo electrónico: enrodiezp@yahoo.es
} 


\section{From subjective aesthetics to interpretative condition: between Kant and Heidegger}

\section{Abstract}

By establishing a relationship between Kant's aesthetics and Heidegger's hermeneutic perspective we can see a rethinking of conception of the world and of the human being that allows establishing similarities and contrasts. The reflections on art in Kant's Critique of Judgment allow seeing cracks in the whole system: the feelings of the beautiful and the sublime by bringing the powers destabilize the system. From Heidegger's point of view these fissures lead to interpretive turn. The world is in a different relationship, without a subject, like a relationship between earth and sky, divinities and mortals. The human being is no longer subject, rests on his own mortality as an event of absence, for no reason. This is a historic shift mode of modernity postmetaphysical rational thought.

Key words: aesthetics, hermeneutics, art, life. 


\section{De l'esthétique subjective à la condition interprétative: entre Kant et Heidegger}

\section{Résumé}

En établissant une relation entre entre l'esthétique kantienne et la perception heméneutique de heideggerienne on percoit une nouvelle interprétation de la conception du monde et l'être humain. Qui permet'de mettre en relief les aspects similaires et les contrastes. Les réflexions sur l'art dans Kant dans son livre "La Critique du Jugement" laisse entrevoir une fissure dnas tout le système: les sentiments du beau et du sublime qui en mettant en jeu les facultés désestabilise tout le système. Du point de vue de Heideger cette fissure nous conduit à une nouvelle tounure interprétative. Le monde 'se transforme en une relation différente entre le ciel et la terre, les divinités et les mortels. L'être humain qui n'est plus un sujet, se soutient dans sa progre mortalité comme un évènement d'absence, san fondement. C'est un mode de déplacement historique de la modernité rationnelle de la pensée postmétaphysique.

Mots clés: Esthétique, herméneutique, art, existence. 


\section{Introducción}

Entre Kant y Heidegger hay un vínculo de diferencias y semejanzas al reflexionar sobre el arte y la obra de arte. En este sentido, el propósito de este texto es comprender, en particular, el ámbito estético en el sistema kantiano para posteriormente desarrollar la crítica de Heidegger a esta visión estética y exponer las diferencias y aproximaciones entre las dos concepciones sobre el arte. Mientras que en Kant se da una experiencia de subjetivización del arte, en Heidegger, por el contrario, hay un encuentro con la obra de arte como acontecimiento de verdad que no es ni subjetivo ni objetivo. Precisamente, este modo de abordar la obra de arte en estos pensadores permite diferenciar y relacionar la visión metafísica y posmetafísica del arte. A su vez, estos dos modos de pensar dejan ver el carácter ambiguo del arte: evento velado y reservado que se origina en una dialéctica o encuentro de fuerzas opuestas que producen sentimientos e ideas inagotables que requieren interpretación.

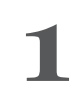

La ambigüedad que es propia de lo estético crea el diálogo entre Heidegger y Kant. Estas han sido dos reflexiones filosóficas sobre el arte, quizá opuestas, pero que se alimentan mutuamente. Ambas apuntan hacia la dimensión de lo sensible y al carácter imaginativo de la obra de arte. En esta interrelación se contraponen dos momentos históricos: la perspectiva moderna en Kant y la visión posmetafísica en Heidegger. Esta discusión bilateral, por tanto, ayudará a comprender mejor el sentido del arte en el mundo contemporáneo.

El desplazamiento del pensar en Kant hacia lo estético puede verse como un acercamiento progresivo a un ámbito en el que un velo indefinible encubre el entendimiento y la razón. En este proceso, la razón teórica y la razón práctica se reexaminan desde la Crítica del Juicio: "El juicio se llama estético también solamente, porque su fundamento de determinación no es ningún concepto, sino el entendimiento (del sentido interno) de aquella armonía en el juego de las facultades del espíritu en cuanto puede ser sólo sentida..." (Kant, 1983, p. 128). El sentimiento de placer y dolor como estado del espíritu se eleva sobre 
los conceptos que unifican lo diverso y sobre las ideas que permanecen en el ámbito suprasensible. Conceptos e ideas no desaparecen, se hacen menos evidentes en la experiencia estética, en el sentimiento que provoca lo bello o lo sublime bajo el juego libre de las facultades. En el ámbito estético se da el encuentro, en la finitud y en lo particular, con la universalidad y la infinitud. De este modo, lo estético conserva su autonomía como esfera particular del pensar. Esta es la visión del arte desde la subjetividad, desde la experiencia interna en el juego libre de las facultades del conocer; experiencia que no se pierde en una simple subjetivización del arte sino que es un reconocimiento de lo propio del arte que mantiene un doble juego de fuerzas, una dialéctica distinta a la dialéctica del entendimiento o de la razón.

Por otra parte, el pensamiento de Heidegger se dirige ya no a la experiencia interna, sino a la obra de arte misma como fuente del pensar. El mismo pensador también se desplazó de una perspectiva antropocéntrica del ser humano, el “Dasein", el ahí del ser, del Sery Tiempo, que es el lugar privilegiado en donde se despliega la pregunta por el ser, hasta una consideración de la obra como evento en el que, en un juego de desocultamiento y ocultamiento, la verdad aparece en una penumbra en la que luz y sombra se encuentran. Pero tampoco se llega a una objetivización de la obra, es decir, a una visión de la obra como mero objeto que afecta los sentidos. Más bien, muestra la constitución de la obra, por un lado, desde su configuración y sus horizontes de comprensión y, por otro, desde la actividad interpretativa que tiene que ver con la experiencia histórica del ser humano cuando se ve interpelado por la obra de arte. Este acercamiento al acontecimiento artístico también pone en evidencia una nueva dialéctica que no es racional ni conceptual, sino, más bien, un encuentro de fuerzas, que crean lo que Heidegger llama Cuaternidad: proximidad de cielo y tierra, de divinidades y mortales. Por tanto, el arte es el acontecer en donde estos cuatro ámbitos se entretejen:

A este juego de espejos de la simplicidad de tierra y cielo, divinos y mortales -un juego que acaece de un modo propio- lo llamamos mundo. El mundo esencia haciendo mundo. Esto quiere decir: el hacer mundo del mundo no es ni explicable por otra cosa que no sea él, ni fundamentable a partir de otra cosa que no sea él. Esta imposibilidad no radica en que nuestro pensamiento de hombres no sea capaz de este explicar ni de este fundamentar. Lo inexpli- 
cable e infundamentable del hacer mundo del mundo se basa más bien en el hecho de que algo así como causas o fundamentos son algo inadecuado al hacer mundo del mundo. Así que el conocimiento humano reclama aquí un explicar, no traspasa los límites de la esencia del mundo sino que cae bajo la esencia del mundo. El querer explicar del ser humano no alcanza en absoluto lo sencillo de la simplicidad del hacer mundo. Los Cuatro, en su unidad, están ya asfixiados en su esencia si nos los representamos sólo como algo real aislado que debe ser fundamentado por los otros y explicado a partir de los otros (Heidegger, 1994, pp. 156-157).

Estas reflexiones muestran una nueva condición del pensamiento que rompe con las limitaciones de la modernidad metafísica; desatan los lazos de la razón instrumental e idealista, centrada únicamente en la universalidad de la lógica que concebía el mundo desde conceptos preelaborados. Ante todo, aquí, el juego de relaciones se moviliza en el tiempo humano mortal, pero en relación con lo sagrado, con la tierra como materia de la naturaleza y con el cielo como aspecto visible de cierta eternidad; mientras que en el pensamiento racionalista, la noción de sujeto reduce estas relaciones tanto que no deja ver cada uno de estos cuatro elementos en su mutuo encuentro. Sin embargo, en la estética kantiana hay cierto resquebrajamiento de esta concepción idealista y subjetiva, de ahí que la intención es mostrar dichos indicios.

Visto así el problema, la lectura de Kant permite reconocer que en su sistema de las tres críticas, en la Crítica del Juicio, el juicio estético crea un ámbito "abierto" para el arte. A pesar de tener una perspectiva moderna centrada en la racionalidad y en la subjetividad, va más allá de su tiempo y, al conceder autonomía al ámbito estético, hace posible que en él se geste con más propiedad el pensamiento contemporáneo de Heidegger, quizá posmetafísico.

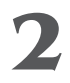

Al construir su sistema, Kant busca la autonomía del arte. Para comenzar, en la Crítica de la Razón Pura, por ejemplo, la relación entre la intuición y el concepto es de carácter universal abstracto; el espacio y el tiempo son categorías universales trascendentales para el conocimiento de objetos. El 
entendimiento unifica la diversidad de las representaciones dadas espaciotemporalmente; sin embargo, lo contingente, lo particular, queda fuera de esta unificación debido a su carácter múltiple y diverso. Por otro lado, las leyes de la naturaleza son la elaboración abstracta de las relaciones necesarias que se dan entre los objetos. El entendimiento tiene un carácter trascendental, universal, lógico y abstracto; por esto, la relación del sujeto con el objeto sólo es fenoménica. El sujeto determina al objeto (como fenómeno), no al noúmeno (la cosa en sí). Se trata de una relación cognoscitiva subjetiva en la que la finitud e inmediatez de las cosas se desvanece, es absorbida por las categorías subjetivas a priori. Precisamente, en la finitud como tal, la fuerza de la infinitud del sujeto sobrepasa los términos particulares de las cosas. De todas maneras, el entendimiento recoge en sí lo intuido; entonces, el entendimiento supera la intuición al poner en juego categorías universales. Pero en este momento teórico algo va quedando por fuera del sujeto, la particularidad escapa de la subjetividad por alguna fisura. Kant no dejará que ello ocurra; por tanto, con la facultad de juzgar, que es particular, en la que ni el entendimiento ni la intuición se condicionan entre sí, cierra el círculo de la comprensión de lo particular. En otras palabras, con el juicio particular lleva a cabo la subjetivización del mundo.

Por eso el juicio debe, para su propio uso, aceptar como principio a priori que lo contingente para la humana investigación en las leyes particulares (empíricas) de la naturaleza encierra una unidad en el enlace de su diversidad con una experiencia posible en sí, unidad que nosotros no tenemos ciertamente que fundar, pero pensable, sin embargo, y conforme a la ley (Kant, 1983, p. 82).

Este ha sido un proceso constante de construcción del ámbito estético. Por esto, la Crítica del Juicio completa el movimiento hacia la subjetividad en el juego espontáneo de las facultades, en el que el arte, como evento particular, se percibe como algo universal.

En la Crítica de la Razón Pura está el origen de la discusión sobre la lógica y el conocimiento, pero la discusión posmoderna afirma que se ha desbordado ese sentido lógico de la modernidad. No sólo la ciencia conoce la verdad de las cosas por su precisión, legalidad y coherencia lógica. Las leyes de la naturaleza, expresadas en el sistema de la matemática y la lógica en su absoluta 
abstracción y universalidad, no determinan ni el ser ni la verdad. Por ello, es necesario identificar en qué medida lo lógico, lo teórico, es decir, el ámbito de la razón pura, puede ser superado por lo estético. En este sentido, el juicio reflexionante de la facultad de juzgar es la raíz de la discusión contemporánea y hace evidente una relación nueva entre lo lógico y lo estético. Esta fisura se pone en evidencia cuando:

(...) bajo estas condiciones, lo que es, sólo puede llegar a ser si cabe en ese restringido territorio de la experiencia. Mas si bien ésta ha ganado, gracias a la matematización, certeza y exactitud, también ha dejado por fuera la complejidad de relaciones en que se hallan los objetos particulares, lo que tienen estos mismos de incierto y azaroso, de inexacto y contingente (Carrillo, 1984, p. 61).

Esta fisura en lo azaroso, en lo inexacto y en lo contingente que Kant elude en su momento teórico mediante los conceptos del entendimiento, va a ser abierta por la Crítica del Juicio. La estética kantiana dará un nuevo sentido a la razón teórica. Y ahora puede decirse que la razón teórica sólo se comprende ampliamente desde la facultad de juzgar porque se torna estética.

El problema estético consiste en cómo establecer la relación del sujeto con el objeto en las condiciones particulares y contingentes de la experiencia humana. Justamente, mediante el sentimiento de placer y de dolor, el ser humano se hace finitamente infinito. Esta era la limitación de la razón teórica:

Como tema central de la Crítica de la Razón Pura, señala Heidegger el problema de la finitud del conocimiento humano. En la formulación de esa finitud y en la permanente conciencia que de ella se tiene, reside según él, la característica decisiva que distingue la teoría de Kant de todos los otros sistemas dogmáticos "precríticos" (Cassirer, 1979, p. 110).

Este es un estado del espíritu que determina el acontecer de la experiencia humana. En consecuencia, la razón teórica toma una dimensión distinta. Es decir, antes de que el entendimiento unifique las percepciones ocurre la experiencia estética. El entendimiento que somete a la intuición en la razón pura, en la facultad estética se constituye como juego libre ante la experiencia de lo bello. Allí, concepto e intuición se aproximan en la apariencia de la forma sin desaparecer uno en el otro. 
En el sentimiento de placer, lo bello deja brotar ese equilibrio; un equilibrio, no individual sino universal, desinteresado, que reconoce una finalidad sin fin, mediante un sentido común. Es decir, la facultad de juzgar cura las deficiencias de la razón teórica. El sujeto, en su facultad universal y abstracta, que posee las leyes necesarias y lógicas del entendimiento, encuentra una experiencia en su finitud: en el sentimiento frente a lo bello, el sujeto reconoce de inmediato ese equilibrio a través de lo sensible. De este modo, el sentimiento crea, desde la particularidad, la relación entre entendimiento e intuición. En esta experiencia estética de lo bello, el ser humano se proyecta en su auténtica dimensión:

Sin embargo, nosotros pensamos que una lectura atenta de la Crítica del Juicio nos permite comprender, más bien, que en la estética de Kant el "sujeto trascendental" se diluye ajustándose a la concordancia entre lo particular y lo universal en la unidad del juicio estético (Carrillo, 1984, p. 65).

El sentimiento que produce lo bello en el sujeto sitúa al ser humano en su contingencia y a la vez en su universalidad. Por eso, ante lo bello sólo se toma una actitud contemplativa que no somete a la obra al interés puramente individual.

La polémica contemporánea entre ciencia y arte se origina en esta relación teórica del conocimiento: la verdad no está sólo en la ciencia; en el arte también la verdad aparece. La estética, en este sentido, es una experiencia más radical que la teoría del conocimiento; más acá de la lógica está la estética. Por tanto, hay que reconocer de qué manera se puede recuperar, de una forma más compleja, la relación entre ciencia y arte desde una mirada menos teórica.

Pero en el movimiento kantiano hacía la razón en la Crítica de la Razón Práctica, de nuevo van a aparecer más limitaciones: "Hay, pues, un campo ilimitado, pero también inaccesible, para nuestra total facultad de conocer; es a saber el campo de lo suprasensible" (Kant, 1983, p. 74). Pero la facultad de juzgar permite también una superación de estos límites de lo finito. Como se ha anotado, la razón teórica es insuficiente cuando se trata de conocer lo particular efectivo; entonces, en el paso del entendimiento a la razón, apa- 
recen las antinomias, una de ellas es la contraposición necesidad-libertad. Las leyes de la naturaleza posibilitan el conocimiento teórico de los objetos, pero ¿cómo es posible comprender lo que no está dominado por esas leyes, por ejemplo, todo el ámbito de la metafísica: las ideas de alma, de libertad y de la naturaleza de Dios? En particular, la idea de libertad, pertenece a lo suprasensible que escapa de las leyes de la naturaleza. Entonces, la idea de libertad se comprende como aquella idea que guía la razón sin ser ella misma la razón; por esto, es una idea caracterizada por una inteligibilidad de lo ininteligible. En consecuencia, la libertad no se puede definir como tal; sin embargo, es una idea que regula los actos humanos.

En lo que se refiere a la idea de libertad y con ello a la misma razón práctica insiste expresamente Kant, en que ella en tanto un puro "inteligible", no está vinculada a simples condiciones temporales. Ella es más bien la pura mirada atemporal, el horizonte de lo supratemporal (Cassirer, 1979, p. 118).

La idea de libertad, por tanto, es una ley moral ante la que se tiene un sentimiento de respeto. De ahí que el actuar humano no se guía por la inclinación sino por el respeto a la ley, por el deber ser. La idea de la libertad se halla, de este modo, entre lo sensible y lo inteligible; he aquí un nivel de ambigüedad que se hará más complejo en el ámbito estético, puesto que la experiencia artística se vincula con la idea de la libertad.

En el entendimiento, el concepto reúne la diversidad que se da en la intuición espacio- temporal; en la razón, la idea de libertad orienta el actuar. Sin embargo, hay una fisura en la razón práctica: no se puede afirmar sin equívocos que es el sentimiento de respeto a la ley lo que ocurre:

Así Kant muestra que el yo no podría adoptar la ley moral de otro modo que como sentimiento de respeto, aparece nuevamente, con esta fundamentación sobre un sentimiento, la vinculación y la finitud y con ella, la relación con la constitución "originaria de la imaginación trascendental" (Cassirer, 1979, p. 118).

El ser humano, entonces, está sometido a la ley moral. ¿Dónde halla su individualidad?, ¿cómo pueden sus circunstancias determinar su moralidad? El ser humano existe en lo contingente; está involucrado con la inmediatez de lo real y lo circunstancial, pero la ley moral kantiana, como deber ser, so- 
mete esta contingencia vital, es decir, la ley moral reprime las inclinaciones y pasiones. ¿Cómo vislumbrar una salida a esta limitación de la moralidad, a esta sumisión a lo bueno, al deber ser, por un lado y al concepto, por otro? De nuevo, la estética kantiana se va encontrando con estas limitaciones y prepara su distanciamiento. El sentimiento o estado del espíritu deja entrever, a través de la experiencia estética, cómo el ser humano tiene vitalmente un encuentro con el objeto en su finitud, desde su sensibilidad. Como sujeto llega a la armonía causada por la contemplación desinteresada de lo bello que le causa placer en lo inmediato; pero, ahora sufre una conmoción interna en sus facultades al padecer lo sublime. Entonces, experimenta en lo particular la idea de libertad que se manifiesta en el sentimiento que produce. Como en la experiencia estética sentimental no hay mediación, el ser humano se encuentra entre lo contingente y particular, sin abstracciones ni juicios teóricos.

De este modo, la facultad de juzgar se vincula con la razón práctica. Lo sublime, entonces, provoca una sensación abrumadora de libertad en la experiencia estética, como si esa idea reguladora rompiera el proceso unificador del concepto y dejara, en medio de lo contingente, el sujeto, a merced de las fuerzas inconmensurables de lo extremadamente grande. Por eso, en el juicio estético la libertad encuentra plenitud: la idea estética, aquello que hace pensar sin ser pensamiento, aparece en el sentimiento de lo sublime, en el abismo que se abre en el terrible juego de la noche y que consume nuestro espíritu en conmoción total.

En la experiencia estética se expresa la totalidad, como si lo universal estuviese contenido en la particularidad del objeto bello. El juego entonces, no sólo es juego subjetivo de las facultades, sino, que también es juego entre el individuo y el conjunto de la realidad. Dado ese libre juego, podemos afirmar también, que el verdadero sujeto de la experiencia estética, según Kant, no es el hombre, sino la profundidad misma de la naturaleza que actúa por medio del "genio", del artista, haciéndolo, como creador de lo bello y de lo artístico, el depositario de sus fines (Carrillo, 1984, p. 170).

Entonces, lo sublime pone en actividad el espíritu, en su particularidad. No se trata del respeto a una ley universal moral. Más bien, sucede el encuentro de sí mismo en su intimidad, en su contingencia, en su particularidad; 
se trata de la experiencia del sentimiento doloroso del estar vivo que no se logra comprender ni con la universalidad del concepto, ni con la absolutez de la razón.

Como se ve, todo el movimiento del pensamiento de Kant conduce a la estética. Por tanto, el sentimiento de placer se relaciona con el juego libre entre entendimiento e intuición y el sentimiento de dolor con el juego libre entre razón y sensibilidad. Así, la experiencia estética ha integrado la facultad del conocer en el sentimiento de lo bello y la facultad de desear en el sentimiento de lo sublime. De esa manera, la estética kantiana genera nuevas reflexiones sobre la comprensión contemporánea del arte y la existencia.

Así como en el primer momento se establece una relación entre ciencia y arte, al considerar la razón práctica surge el problema de cómo ética y arte pueden relacionarse. Esto también determina las diferencias entre la modernidad y el pensamiento posmetafísico. La crítica posmoderna pone en entredicho el carácter universal de la ley moral, precisamente de origen kantiano, que concibe que el deber ser somete el actuar a unas reglas, a un criterio que dice qué es bueno y qué es malo. Nietzsche desborda esta visión y muestra que hay un ámbito de lo ético que supera la dicotomía excluyente de lo bueno y lo malo y es, precisamente, lo artístico. De igual modo, Heidegger anula las diferencias entre ética y estética y su perspectiva se dirige al "ser" que acontece en la obra.

Bajo esta mirada, la existencia se torna estética; la vida es como una obra de arte. Esto prueba que el mismo pensamiento de Kant al vincular la dimensión estética con la ética, con la idea de libertad, genera otras miradas. Lo más libre, no está en la moralidad, en la idea práctica de la libertad, sino en la experiencia sensible e imaginativa que se produce en Facultad de Juzgar al considerar la obra de arte. Por tanto, la estética alcanza un nivel de autonomía que desborda una ética de los deberes; en consecuencia, la ética y la estética establecen una relación distinta, en la que se complementan.

En este momento estético, en esta agitación interna, en la experiencia subjetiva del sentimiento de lo bello y de lo sublime, el hombre es auténticamente libre en su finitud y contingencia, porque en su particularidad, en sus 
circunstancias, experimenta lo bello o lo sublime, desde la individualidad en la que algo particular, bello o sublime, afecta su experiencia instantánea. Una obra particular, un acontecimiento individual y contingente, en la finitud de sus circunstancias (el color, la posición en el espacio, el sonido, la palabra) afectan sensiblemente al ser humano en su condición finita. De este modo, se han superado las fisuras que aparecían en la razón teórica, debido a su carácter abstracto, y en la razón práctica, debido al modo como el sentimiento moral de respeto se convierte, de igual forma, en una manera abstracta de asumir la ley moral.

La estética kantiana, como experiencia subjetiva, permite descubrir que la vida, antes que depender de los conceptos del entendimiento, de la lógica, de la verdad de la ciencia o antes que estar sometida a la ley moral, es una experiencia que surge de las fuerzas de la inmediatez y acontece en un encuentro, activo en lo sensible y en la finitud, con el mundo y sus concepciones. En consecuencia, la vida se funda en la actitud estética. De ahí que se pueda inferir que la estética es un ámbito más radical que lo teórico y lo práctico porque el sujeto en su individualidad y particularidad finita, con sus sentimientos y su sensibilidad, reconoce su universalidad e infinitud como propiedad de lo humano. Hay que observar que en Kant la relación entre finitud e infinitud queda abierta, a diferencia de la dialéctica que más tarde sistematizará y cerrará Hegel. Como se ve, en esta concepción se muestra el carácter ambiguo del arte que juega libremente con la facultad teórica y la facultad práctica sin que ninguna se imponga sobre la facultad estética. Es decir, el arte provoca un juego entre la sensibilidad y el concepto y entre el mundo sensible de las inclinaciones y el mundo inteligible de la libertad. En medio de esas dimensiones se sitúa el conocer sin conceptos debido a la sensible inteligibilidad que ocurre en la obra de arte. De ahí que Kant se vincule necesariamente con las estéticas contemporáneas que han reconocido lo primordial de la dimensión estética.

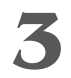

Es posible mirar este avance de Kant hacia la estética desde la perspectiva de Heidegger para reconstruir un camino inverso. Indudablemente que 
para Kant el arte es una experiencia que se da en la subjetividad. Su estética describe cómo las facultades entran en un juego libre en el sujeto. Heidegger da un giro radical frente a esta estética idealista de la subjetividad que recibe en sí todo acontecer exterior. La nueva actitud considera la obra de arte como ámbito de verdad y al ser humano como alguien que existencialmente experimenta la obra en su darse, sin llegar a establecer categorías ni subjetivas ni objetivas que expliquen la obra artística.

Heidegger hace una crítica al temor de Kant para afrontar el abismo que se pierde en lo profundo del alma: "Lo que él quiere mostrar es precisamente esto: que Kant, después de haber logrado descubrir la raíz común del 'entendimiento'y desenterrar la 'sensibilidad' de la imaginación trascendental, habría retrocedido ente su propio descubrimiento" (Cassirer, 1979, p. 123). Este es el abismo de la temporalidad que la imaginación constituye. En la sucesión de las diversas representaciones que se dan en la intuición se despliega el tiempo, no como categoría trascendental sino como evento de la finitud humana. En ese despliegue del tiempo, que en cierta forma también es espacial, surge la dimensión de la mortalidad, que no es categoría de la metafísica de lo suprasensible, sino condición propia del hombre. Kant retrocede ante este abismo, por ello retorna a la subjetividad.

En este camino reflexivo, Heidegger da un giro hacia el afuera del sujeto. Reconoce que en esa finitud abismal, que no alcanzó a abordar plenamente Kant, acontece la obra de arte. Para el pensador metafísico, en la imaginación trascendental, receptiva y dinámica se encuentran lo universal, lo particular, lo abstracto y también lo sensible, lo finito y lo infinito. Por medio de ella, el sujeto tiene experiencia del mundo en sus determinaciones inmediatas, en una obra concreta, mediante sus características sensibles: color, forma, sonido. La totalidad de lo bello y lo sublime provocan el estado sentimental e imaginativo: "Ella (la finitud) se manifiesta en el hecho de que todo el conocimiento humano depende de sus intuiciones y que toda intuición es 'receptiva'y consiste en una'recepción' originaria" (Cassirer, 1979, p. 110). En este punto no habría muchas diferencias entre los dos pensadores.

Para Kant, en el acontecer del sentimiento de placer y dolor, en un instante, la eternidad abruma al ser humano, mientras que en la unificación en el con- 
cepto del entendimiento o en el carácter regulativo de la razón sólo se pone en juego la dimensión abstracta del ser humano. Para Heidegger, en este estar en el mundo, sintiéndolo, el ser humano se desvanece, se constituye en su "ser-para-la-muerte". De hecho, la concepción kantiana de la muerte tiene una perspectiva metafísica, mientras que en Heidegger es un estado de ser que es propio de su condición de arrojado. Ahí comienza el distanciamiento entre el esteta y el hermeneuta.

Kant describe lo que pasa dentro del sujeto respecto de lo bello y lo sublime. Heidegger mira lo bello y lo sublime en la obra, sin necesidad del sujeto. Sin embargo, los dos dejan un margen de libertad a la dimensión artística: Kant no se atreve a definir la indefinibilidad de las ideas estéticas; Heidegger no afirma que la verdad se aclara en la obra, al contrario, dice que la obra oscurece, en su claridad, la verdad.

Como se ha dicho, a partir de Kant y Heidegger el arte se comprende desde su carácter ambiguo porque produce una tensión entre dos fuerzas, un encuentro de dos direcciones que configura un estado de indefinibilidad: sensibilidad y razón conjugados para Kant; cielo y tierra, divinidades y mortales para Heidegger. Esta última relación es la manifestación de la dialéctica estética y la lucha entre las cuatro dimensiones que sucede de manera abierta en la obra de arte; en ella, el decir y el ocultar, el sentir y el pensar se aproximan pero mantienen sus diferencias. Este juego es múltiple, diverso y dinámico. Esta tensión nunca se resuelve, sólo la experimenta intuitivamente el ser humano: “La vinculación originaria a la intuición no puede ser nunca anulada y no es posible superar la dependencia que ella establece. La cadena de la finitud no puede romperse" (Cassirer, 1979, p. 111). De ahí que el ser humano sea el lugar de apertura del ser, que borra la sustancia subjetiva. Lo bello, lo sublime deja de ser una percepción subjetiva porque destruye la dicotomía sujeto-objeto. La obra se da al intérprete y a la vez el intérprete se abre a la obra.

Al desaparecer los conceptos de sujeto y objeto, se constituye una región imprecisa en la que el espectador, desde su perspectiva y su entorno próximo, entra en contacto con la obra; se vuelve lector que ha sido transformado por la obra, que toma distancia de la realidad, pero sin alejarse de ella. Por el 
contrario, se ve conducido a entrar en diálogo histórico con su tiempo al ser tocado por la cercanía de las cosas. Como Heidegger lo indica, esta actividad interpretativa es posible por el poetizar que es un modo de habitar el mundo.

De este modo, estamos ante una doble exigencia: primero, pensar lo que denominamos la existencia del ser humano desde la esencia del habitar; luego, pensar la esencia del poetizar en tanto que dejar habitar como un construir, incluso como el construir por excelencia. Si buscamos la esencia de la poesía desde la perspectiva de la que acabamos de hablar, llegaremos a la esencia del habitar (1983, p. 165).

De manera que Heidegger, al exceder la dicotomía platónico-kantiana de sujeto y objeto, se encuentra con que el ser humano vive arrojado al mundo y es de suyo morirse; es un Dasein, un ser ahí, también ambiguo como la obra de arte porque se sostiene entre las fuerzas del mundo y determinado por sus circunstancias, pero sosteniéndose entre su autonomía y lo contingente.

Esta relación hasta ahora expuesta es importante para la discusión sobre las relaciones entre ciencia, ética, política y estética en la actualidad. Se puede inferir, por tanto, que la ciencia puede vincularse con el arte, la ética puede ser estética, según el recorrido que hace Kant; o desde el punto de vista heideggeriano, la verdad y el ser no sólo se dan en la ciencia, o en la moralidad, sino también, y con más intensidad, en la obra de arte, debido a su carácter ambiguo y sobre todo porque abren espacio al espectador, al lector o al interprete, para que habite este mundo de una manera distinta al estar sometido al mundo de la objetividad que se ha reducido a un trato utilitario y comercial con las cosas. En fin, creo que la reflexión sobre lo estético y el arte ofrece mayores posibilidades que aún están por precisarse a partir de estas reflexiones iniciales.

En síntesis, cuando Kant y Heidegger conciben el arte a partir de su ambigüedad, se pone en evidencia un cambio en la concepción del ser humano y del mundo que afecta los modos de interacción social y de concepción de la realidad. El sujeto kantiano queda borrado por la transformación del sentido del ser que se devela en la obra de arte. Pero al reconocer el papel de la facultad estética de Kant, es posible este desbordamiento. 
La obra de arte, como evento de verdad, como luz en mitad del bosque, desestructura todo lo estable que el pensamiento metafísico logró construir. La obra como juego entre develamiento y ocultación desplaza la luz de la razón y provoca una ambigüedad que equivale al ámbito de lo poético. Desde esta perspectiva, el sujeto también es afectado por dicha ambigüedad; por tanto, debe ser concebido como evento del ser, como aparición que se muere, como ser que habita poéticamente esta tierra, en palabras de Hölderlin y que evoca Heidegger persistentemente. Queda en el eco de estas palabras de Heidegger la huella de la ausencia que trasluce la muerte, lo sin fundamento, el siempre estar en el mundo pleno de vida y de muerte:

El ahí "es" el hombre sólo como histórico, es decir, fundador-de-historia y con instancia en el ahí en el modo del abrigo de la verdad del ente. Sostener el ser-ahí sólo con instancia en el recorrido creador más elevado, es decir, a la vez sufriente, de los más amplios éxtasis. Al ahí pertenece como su extremo esa ocultación en su abierto más propio, lo ausente, como permanente posibilidad el estar-ausente; el hombre lo conoce en las diferentes figuras de la muerte. Pero donde ser-ahí ha de ser concebido por primera vez, tiene que estar determinada la muerte como extrema posibilidad del ahí (Heidegger, 2003, p. 263).

\section{Referencias}

Cassirer, E. (1979). El problema del conocimiento en la filosofía y en la ciencia modernas (vol. 4). México: Fondo de Cultura Económica.

Carrillo, L. (1984). El problema de la experiencia estética en Kant. Ideasy Valores. Bogotá: Universidad Nacional de Colombia.

Heidegger, M. (1954). Kant y el problema de la metafísica. México: Fondo de Cultura Económica.

Heidegger, M. (1983). Interpretaciones sobre la poesía de Hölderlin. Barcelona: Ariel.

Heidegger, M. (1996). Conferencias y artículos. Barcelona: Odos. 
Heidegger, M. (2003a). Aportes a la filosofía. Acerca del evento. Buenos Aires: Biblos.

Heidegger, M. (2003b). Ser y tiempo. Madrid:Trotta.

Kant, E. (1983). Crítica del juicio. Observaciones sobre lo sublime y lo bello. México: Porrúa.

Kant, E. (1985). Fundamentación de la metafísica de las costumbres. México: Porrúa. 